\title{
Diacronie
}

Studi di Storia Contemporanea

$\mathrm{N}^{\circ} 25,1 \mid 2016$

"Se creare è definire"

\section{Alba Mora (a cura di), Il Collegio dei Nobili di Parma. La formazione della classe dirigente (secoli XVII-XIX)}

\section{Alessandro De Luca}

\section{(2) OpenEdition}

Journals

\section{Edizione digitale}

URL: http://journals.openedition.org/diacronie/3886

DOI: $10.4000 /$ diacronie.3886

ISSN: 2038-0925

Editore

Association culturelle Diacronie

Notizia bibliografica digitale

Alessandro De Luca, «Alba Mora (a cura di), Il Collegio dei Nobili di Parma. La formazione della classe dirigente (secoli XVII-XIX) », Diacronie [Online], Nㅜ25, 1| 2016, documento 11, Messo online il 29 mars 2016, consultato il 23 septembre 2020. URL : http://journals.openedition.org/diacronie/3886 ; DOI : https://doi.org/10.4000/diacronie.3886 


\section{RECENSIONE:}

\section{Alba MORA (a cura di), Il Collegio dei Nobili di Parma. La formazione della classe dirigente (secoli XVII-XIX), Parma, MUP, 2013, 394 pp.}

a cura di Alessandro DE LUCA *

Il libro in oggetto tratta il tema della formazione della classe dirigente del Ducato farnesiano, uno degli Stati di antico regime più importanti d'Italia, soprattutto per le innovazioni in campo culturale, politico, sociale e amministrativo che in esso si produssero durante l'età del riformismo illuminato a metà del Settecento.

A tal proposito emerge con chiarezza che il libro, frutto degli atti del Convegno nazionale tenutosi a Fornovo, Sala Baganza e Fontevivo tra il 22 e il 24 maggio del 2008, ed edito a Parma nel 2013 da MUP - una diretta emanazione della Fondazione Monte Parma -, non è un testo di storia locale ma una serie di contributi che a partire dal 1601 fino ad arrivare al 1831 tocca i temi più importanti che interessarono il funzionamento e l'organizzazione di un tipico Stato dell'Ancien Régime passato attraverso le tempeste del riformismo illuminato, dell'età napoleonica e della Restaurazione. L'opera si compone di un'introduzione della Prof.ssa Alba Mora, seguita da un contributo di carattere generale di Giuseppe Papagno e da una serie di saggi articolati in tre blocchi tematici: "Gli spazi e i luoghi"; "Politica, istituzioni e società"; "Insegnare ed imparare". In tutto l'opera è consta di 15 saggi. Prima di analizzarli uno ad uno sarà opportuna una contestualizzazione storica del Ducato di Parma e Piacenza.

Il periodo 1601-1831 è stato quello in cui fu creato ed operò il Collegio dei Nobili di Parma con il precipuo fine di educare la futura classe dirigente del Ducato farnesiano: esso tocca quindi anche il tema del rapporto tra élites e masse, per quanto queste ultime si siano affacciate sul palcoscenico della storia europea solo dalla Rivoluzione francese in poi. Essa fece sentire i suoi effetti a Parma ed in Italia dal 1796 in poi, 
interessando lo stesso Collegio, completamente rivoluzionato dai francesi nel $1804 \mathrm{e}$ chiuso dal 1806 fino alla fine dell'età napoleonica per via del boicottaggio che contro di esso fu effettuato dalle nobili famiglie parmigiane, restie ad accettare il nuovo ordine di cose imposto con mano ferma da Napoleone a tutti i territori del suo impero, inclusa, ovviamente, Parma.

È stata la Prof.ssa Alba Mora, già docente all'Università di Parma fino ad anni recenti - prima di Storia degli antichi Stati italiani e poi di Storia del Risorgimento - a dare impulso alle ricerche storiche sul Collegio dei Nobili, e (anche attraverso la storia di questa istituzione educativa) sul travagliato periodo che interessa la storia parmense nel XVII-XIX secolo. Gli strumenti che hanno dato respiro nazionale ed internazionale a queste ricerche sono stati il Convegno internazionale di studi di Fontevivo nel 2003 (su cui torneremo in seguito) e poi quello già citato tenutosi in varie sedi del parmense nel 2008. La nascita e il destino del Ducato di Parma e Piacenza sono direttamente connesse all'importante famiglia dei Farnese prima (1545-1731) e alla dinastia borbonica (1748-1802) poi. Il Ducato fu studiato a tavolino da Papa Paolo III Farnese che, in ossequio alla politica nepotistica largamente praticata nella Chiesa fino al Concilio di Trento, voleva creare uno Stato per il figlio Pierluigi. Il pontefice, grazie alla benevolenza di Carlo V, con la bolla del 26 agosto 1545 istituì il nuovo Ducato ${ }^{1}$ con capitale Piacenza, in cui il primo duca Farnese trovò la morte già nel 1547, al tempo dell'occupazione spagnola della città. In seguito a questo fatto la capitale fu trasferita a Parma, mentre Piacenza fu destinata al ruolo di avamposto militare. Ancora oggi funzioni e strutture delle due città, così diverse tra loro, riflettono bene la differente storia che le ha accompagnate dal Cinquecento all'Unità d'Italia. Nato come frutto della volontà papale e dei suoi rapporti - a volte di collaborazione, a volte conflittuali - con le potenze europee francese e spagnola al tempo in lotta per l'egemonia europea e sulla penisola italiana, il Ducato fu sempre guidato da personaggi di primo piano, in grado di rapportarsi con i potentati europei e di barcamenarsi nel complicato gioco politico e militare che segnò le sorti dell'Italia ai tempi delle signorie e della preminenza spagnola sulla penisola, sancita dalla pace di Cateau-Cambrésis del 1559 e destinata a durare fino all'inizio del XVIII secolo.

Oltre a quello cinque-seicentesco, un altro periodo importante nella storia del Ducato, che lo pose all'avanguardia nella lotta che l'Illuminismo aveva dichiarato all'alleanza tra trono e altare a metà del '700, fu rappresentato dal periodo di governo di Du Tillot, tra il 1759 e il 1771. Al vertice del Ducato dal 1748 fu insediato, a seguito della pace di Aquisgrana del 1748 che chiuse la guerra dei sette anni, Filippo di

${ }^{1}$ TOCCI, Giovanni, Il Ducato di Parma e Piacenza, Torino, UTET, 1987. 
Borbone, esponente della più importante famiglia regnante europea che governava sulla Spagna, sulla Francia e sul Regno di Napoli. La dimensione europea della politica ducale era quindi garantita dai legami di sangue che i duchi potevano vantare con mezza Europa, il che non mancò di far sentire la sua influenza nel piccolo Ducato. Esso accolse il verbo illuminista, non tanto per effetto della propaganda di filosofi ed enciclopedisti, destinata del resto ad attecchire poco in un contesto socio-economico particolarmente arretrato e limitato dalle sue stesse ristrette dimensioni. A livello diplomatico e politico fu necessario per Filippo conformare la sua politica a quella degli altri membri regnanti della sua casata, il che comportò l'adozione dell'anticurialismo, la riorganizzazione amministrativa, l'impegno dello Stato al posto della Chiesa nel settore della formazione, l'adozione di misure economiche tese a smuovere il mondo feudale e ad incentivare l'economia di mercato che si ritrovano nei principali stati europei del tempo.

Soprattutto nei rapporti tra Stato e Chiesa la politica di Du Tillot fu particolarmente incisiva: furono attaccati i privilegi del clero, tolte molte competenze legali ai tribunali ecclesiastici e soprattutto furono espulsi i Gesuiti da Parma nel 1768². Tutti questi provvedimenti si intrecciano strettamente con la storia del Collegio dei Nobili, sia per la preminenza che esso aveva nell'ambito della formazione della classe dirigente parmense, sia perché esso era stato retto fin dalla fondazione, nel 1601, dai Gesuiti. Essi erano l'ordine militante della Chiesa controriformistica, che più di ogni altro dedicò la propria missione evangelizzatrice alla formazione e, anche per questo, furono presi di mira dall'assolutismo illuminato europeo di metà Settecento. Esso riuscì ad espellere l'ordine di Ignazio da Loyola dai principali stati europei negli anni Sessanta, fino ad obbligare il Papa a scioglierlo nel 1773, prima che esso rinascesse all'indomani della chiusura dell'avventura napoleonica nel 1814. In molti Stati il posto dei Gesuiti fu preso dai formatori laici, ma ciò non era possibile a Parma, dove mancava una borghesia produttiva ed intellettuale in grado di assolvere questo compito e dove la nobiltà terriera era nota per l'assenteismo dalle sue proprietà e per lo stile di vita incentrato sulla pura rendita di posizione, in maniera non dissimile da quanto i nobili coevi facevano a Versailles alla corte di Luigi XV.

Il Du Tillot pensò quindi di affidare la guida del Collegio ai padri scolopi, più duttili politicamente, ma non all'altezza del compito che era stato loro affidato: già nel 1772 furono rimossi dopo avere iniziato a far decadere la prestigiosa istituzione d'età farnesiana. Tra il 1773 e il ventennio successivo il Collegio fu gestito dal clero secolare fino al 1792, quando il Duca Don Ferdinando richiamò (unico in Europa) i Gesuiti nel 
suo Stato. Essi, sotto mentite spoglie, tornarono a guidarlo fino a quando l'arrivo dei francesi portò, ad inizio Ottocento, ad una nuova cacciata dei Gesuiti e, nel 1806, alla laicizzazione del Collegio. Venne allora boicottato dalle famiglie parmensi e destinato a rimanere chiuso fino al 1816. Dal 1815 la nuova duchessa di Parma, Maria Luigia d'Austria, già consorte di Napoleone, guidò la Restaurazione in modo moderato nel piccolo e ricostituito Ducato. Affidò la rinascita del Collegio ai padri benedettini, fino alla fusione, avvenuta nel 1831, con il borghese Collegio Lalatta che mise la parola fine al Collegio dei Nobili. Il nuovo istituto in cui élite borghese e nobiliare si fusero 3 prese il nome di Maria Luigia, è ancora operante, e costituisce una delle strutture di formazione più in vista della Parma del XXI secolo.

Terminata questa panoramica analizziamo i singoli contributi del libro. A livello di analisi generale si colloca il saggio di Giuseppe Papagno, che ripercorre in breve la storia del Collegio e si sofferma sull'importanza che le istituzioni culturali hanno avuto nella formazione della classe dirigente, ieri come oggi. Secondo l'autore l'unità culturale dell'Occidente viene rotta tra il 1517 e il 1648, quando nasce e si consolida in Protestantesimo. Il tentativo unitario restauratore portato avanti dai Gesuiti riuscì nei soli paesi cattolici, per cui all'educazione unitaria della società si sostituiscono scuole specialistiche che tramandano origini e riferimenti culturali cattolici destinati alle élites sociali in formazione, ormai banditi dai protestanti. Secondo Papagno quello gesuitico è stato l'ultimo insegnamento olistico, cui poi sono seguiti sistemi settoriali di tipo filosofico (Hegel, Marx) o economico (Malthus, Smith) che hanno avuto la pretesa di essere onnicomprensivi, con il risultato di portare al totalitarismo novecentesco. La sezione "Gli spazi e i luoghi" si apre con il saggio di Carlo Mambriani denominato Creazione e crescita dell'alveare delle nobili api, in cui si spiega come le varie sedi in cui si è svolta l'azione del Collegio riflettono gli ambivalenti rapporti tra gesuiti e corte ducale. All'appoggio incondizionato dei Farnese, segue il progressivo distacco dei Borbone dai Gesuiti, solo in parte recuperato dalla politica reazionaria di Don Ferdinando. Secondo l'autore la rottura dell'alleanza tra trono a altare verificatasi ai tempi di Du Tillot, cui seguì la politica ondivaga e contrastante dei decenni successivi, ha contribuito a determinare la decadenza dell'Istituto. Il saggio di Dario Costi si focalizza sull'architettura del Collegio dei Nobili, legandola alla storia ed alla funzionalità che da essa emana. A tal proposito l'autore propone un percorso didattico, atto a rivalorizzare i luoghi del collegio come fonte storica di conoscenza degli aspetti

\footnotetext{
3 Questo era un segno evidente del vento di rinnovamento, che del resto aveva sempre soffiato sul Ducato da metà Settecento, con la sola notevole eccezione del governo clericale di Don Ferdinando tra il 1765 e il 1802.
} 
socio-economico-culturali delle élites ducali tra XVI ed inizio XIX secolo. Alessandro Campanini con il suo contributo Residenze di villeggiatura dei collegi gesuitici di Parma: Valera, Carona, Villetta e Sala analizza l'impatto dell'Istituto su tutto il territorio ducale, date le numerose sedi che in momenti coevi e diversi hanno scandito la vita del Collegio. Molte di queste erano pensate per l'otium degli ordini religiosi.

Gli stessi programmi di studio dei gesuiti prevedevano l'alternanza tra lavoro, studio e svago come strada maestra per il raggiungimento del miglior equilibrio psicofisico. Le residenze fuori Parma erano quindi destinate non solo agli insegnanti, ma anche agli studenti, che invece trascorrevano il giorno di riposo settimanale (il giovedì) e le ferie per proprio conto, in un quadro delle attività in cui le ferie autunnali si protraevano da fine agosto a fine ottobre. Cristina Cecchinelli studia Le "vacanze grandi" a Fontevivo tra $i$ Farnese e i Borbone soffermandosi sul fatto che in questo paese gli studenti trascorressero parte delle loro vacanze - tra il 1728 fino ai primi anni dell'Ottocento -, in un complesso ducale mai donato ai Gesuiti. Al suo interno lo svago era organizzato attraverso giochi, passeggiate, lezioni di ballo, di musica e di caccia. L'obiettivo era infatti quello di trasformare i rampolli dell'aristocrazia parmense non solo in colti e futuri funzionari, ma anche in cavalieri cristiani a tutto tondo. Anche in vista di quest'obiettivo durante le ferie molto tempo era comunque dedicato alle funzioni religiose.

Anna Còccioli Mastroviti si concentra su un tema archivistico, trattando le nuove acquisizioni documentarie per le residenze di villeggiatura dei Gesuiti nel Ducato, frutto recente delle attività di ricerca dispiegatesi in questo campo nell'ultimo decennio. La seconda parte del libro "Politica, istituzioni e società" si apre con il saggio di Flavio Rurale dal titolo Gesuiti, ceto nobiliare e corti principesche tra XVI e XVIII secolo. In esso si parla della linea politica gesuita, che mirava a fare proseliti grazie alla preparazione culturale dei suoi membri e al ricorso a pratiche sincretiche rispetto alle culture lontane da quella giudaico-cristiana. Il buon gesuita doveva essere confessore, teologo, consigliere e precettore della classe dirigente, in un quadro di adesione dell'ordine ai valori aristocratici dell'Ancien Régime, retto dallo studio e dalle opere , più che dalla contemplazione come via per la salvezza, tanto cara ai giansenisti. Gli stessi sovrani moderni ricorsero volentieri all'apporto dei Gesuiti, anche per scansare le sirene della prassi politica suggerita da Machiavelli. Anche Miriam Turrini analizza il rapporto tra Farnese, Gesuiti e nobiltà, nel quadro di un Collegio la cui funzione era quella di legittimare il potere dei duchi agli occhi dei regnanti europei, in quanto luogo di alta formazione della futura classe dirigente. Non a caso tra le materie più importanti vi erano la storia, la geografia, la filosofia e la teologia. Sergio di Noto Marrella si 
sofferma sul rapporto tra il Collegio e l'Università di Parma: le istituzioni si erano sviluppate con il fine di tutelare e formare i membri di una stessa classe sociale privilegiata, evolvendosi da struttura spontanea in organismo legale controllato dal potere. A Parma il Collegio fu collegato agli insegnamenti universitari, secondo la divisione tra discipline laiche ed ecclesiastiche in vigore al tempo, e poteva essere frequentato da chi aveva tra i dieci e i vent'anni. Dal 1768 l'Università fu rilanciata, mentre il Collegio dei Nobili, privato dei Gesuiti, decadde. Questo rilievo introduce al saggio di Giovanni Gonzi, che riporta alla luce uno dei momenti di rottura più importanti per la storia del Ducato, ossia la cacciata dei Gesuiti da Parma, voluta da Du Tillot nel 1768. Essa ebbe importanti ricadute su tutto il tessuto sociale ducale ed ovviamente anche sullo stesso Collegio, che si trovò privato della sua guida storica. La svolta di Du Tillot non era dovuta a questioni interne: si trattava di allineare la politica del Ducato al giurisdizionalismo in vigore nei principali regni borbonici europei. Ciò portò ad affidare il Collegio ai padri Scolopi, che in breve furono sostituiti da preti secolari, prima del ritorno dei Gesuiti sotto mentite spoglie, deciso nel 1792 da Don Ferdinando. Definitivamente estromessi da Napoleone, il Collegio fu prima laicizzato negli anni francesi e poi affidato ai benedettini nel suo ultimo quindicennio di vita (1816-31).

L'ultimo saggio della seconda sezione, di Michele Basile Crispo, illumina la figura di Scipione Maffei ed in particolare riporta di una beffa da lui compiuta ai danni del duca farnese. Dall'aneddoto si parte per tracciare il quadro dei migliori intellettuali, di caratura nazionale ed internazionale, che sono in parte o in tutto debitori a Parma per la loro formazione.

La terza sezione "Insegnare ed imparare" si concentra maggiormente sugli aspetti didattici e formativi sviluppatisi all'interno del Collegio, con l'intento di collegare l'organizzazione degli studi alla conseguente visione del mondo che emanava dall'ordine gesuitico e dal potere ducale. Marzia Giuliani si sofferma su I libri spirituali e la formazione dei cavalieri cristiani nel periodo 1688-1708. Dagli inventari del Collegio si ricava che in questo periodo i testi più in uso erano di tipo sacro e liturgico. Non mancavano sermoni clericali e moraleggianti, legati alla temperie controriformistica del Seicento e ai suoi più famosi predicatori come Paolo Segneri. Le attività di lettura erano spesso svolte in comune e ad alta voce; questo metodo decadde solo con la rimozione dei gesuiti dal Collegio nel 1768. Giuseppe Martini porta alla luce il rapporto tra la didattica dell'Istituto e la musica, che fu una disciplina per molti versi snobbata. L'opera musicale fece parte dei programmi del Collegio per soli due decenni ed in poche occasioni, coincidenti con il debutto del melodramma sul proscenio 
parmense. Nel Collegio la musica veniva insegnata spesso da docenti esterni, in corsi divisi per strumento, tra cui prevalevano quelli a corde. La musica era del tutto aliena alla cultura dei gesuiti e si diffuse limitatamente nel Collegio solo a metà Seicento, insieme alle rappresentazioni teatrali in cui si alternavano recitazione, intermezzi musicali e danze. Giovanni Zanlonghi analizza un documento del primo Settecento in cui un docente-sacerdote sostiene il diritto del discente di scegliere se recitare o no, a proposito di discipline considerate minori nel Collegio, eppure giudicate attraenti da alcune delle menti più avvertite tra gli studenti dell'Istituto.

Rosa Necchi concentra la sua attenzione sul tema Goldoni, Bettinelli e il Collegio, ricollegandosi al saggio di Crispo, in quanto ha in comune con esso il tema delle migliori menti del Sei-Settecento formatesi anche nell'ambiente parmense. Tra queste viene ricordato Goldoni, che in una sua commedia tesse l'elogio del Collegio di Parma e della sua capacità di formare al meglio i rampolli delle classi dominanti.

Il quadro della formazione all'interno del Ducato non sarebbe completo senza uno studio di quanto è avvenuto a Piacenza, specie nel Settecento. Il saggio di Ugo Baldini si intitola L'altro polo dell'istruzione superiore nel Ducato borbonico: l'insegnamento delle scienze nel Collegio Alberoni (1751-1810). Nella seconda metà del Settecento, proprio mentre a Parma iniziavano le traversie che avrebbero portato alla decadenza del Collegio, cresceva la reputazione del Collegio Alberoni come luogo di formazione alternativo e serio della classe dirigente ducale. Esso era autonomo dal vescovo e retto dall'ordine vincenziano. Il 30\% dei suoi studenti non diventava sacerdote, ma parte della classe dirigente in tutto il Nord Italia. A livello filosofico, nel Collegio Alberoni penetrò il giansenismo, nemico giurato dei Gesuiti e assai sospetto agli occhi delle corti di Ancien Régime, in quanto imputabile di essere molto vicino alle istanze illuministiche, anche di tipo radicale. Il Collegio Alberoni costituì la sua specificità formativa puntando sull'insegnamento delle scienze, dotandosi di laboratori che lo posero all'avanguardia nella formazione scientifica italiana. Questa natura non fu repressa ma incentivata dai francesi dominatori, tanto che da Moreau De Saint Mery in poi le dotazioni dei laboratori crebbero fino al 1815. Dal punto di vista del patrimonio bibliotecario si evince anche come il Collegio Alberoni seppe innovare il suo parco-libri mostrandosi attento ai progressi scientifici di fine Settecento e inizio Ottocento e generando una formazione al passo con i tempi, tumultuosi ma innovativi, che l'età napoleonica stava per innestare in tutta Europa. Questo importante libro è destinato agli studiosi di storia locale ma anche ai cultori della storia moderna, data la dimensione europea di potere che riguardò le famiglie regnanti nel Ducato sia prima che dopo l'età napoleonica, durante la quale, del resto, Parma e Piacenza furono 
direttamente annesse al vasto Impero francese. Il volume costituisce una valida prosecuzione del revival di studi storici fioriti nell'ultimo quindicennio specie sulla fase post-illuminista del Ducato, fino a quel momento trascurata dagli storici, principalmente a causa del carattere anacronistico della politica seguita da Don Ferdinando a fine Settecento. Inoltre il volume ha il merito di essere da un lato un lavoro settoriale e tematico e, dall'altro, un'opera di ampio respiro, che tratta una larga parte della storia del Ducato, dilatando lo spettro cronologico degli studi ad esso dedicati. La potenzialità più importante dell'opera consiste infatti nel legare visione storica locale e globale dei secoli oggetto di studio, aprendo nuove possibilità di ricerca che sappiano legare temi di vasta portata assieme all'analisi dettagliata di realtà circoscritte, ora ben note grazie al rigoroso lavoro di ricerca e interpretazione delle ampie fonti archivistiche disponibili. 


\section{* L'autore}

Alessandro De Luca, nato nel 1979, è docente di materie storiche e letterarie presso le scuole medie superiori. Addottoratosi in Storia presso l'Università di Parma nel 2012, ha scritto vari saggi e monografie sull'età napoleonica in Italia, trattata dal punto di vista politico ed economico. Tra i suoi interessi di ricerca relativi alla storia del novecento vi è il socialismo autogestionario.

URL: < http://www.studistorici.com/progett/autori/\#DeLuca >

\section{Per citare questo articolo:}

DE LUCA, Alessandro, «Recensione: Alba MORA (a cura di), Il Collegio dei Nobili di Parma. La formazione della classe dirigente (secoli XVII-XIX), Parma, MUP, 2013, 394 pp.», Diacronie. Studi di Storia Contemporanea : "Se creare è definire", 29/03/2016,

URL:<http://www.studistorici.com/2016/03/29/deluca_numero_25/ >

Diacronie Studi di Storia Contemporanea $\}$ www.diacronie.it

Risorsa digitale indipendente a carattere storiografico. Uscita trimestrale.

redazione.diacronie@hotmail.it

Comitato di redazione: Jacopo Bassi - Luca Bufarale - Elisa Grandi - Antonio César Moreno Cantano - Deborah Paci - Fausto Pietrancosta - Alessandro Salvador - Matteo Tomasoni - Luca Zuccolo

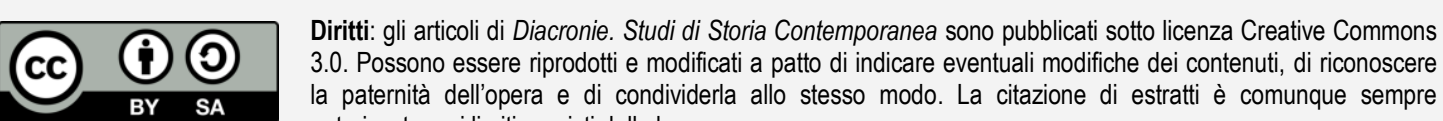
autorizzata, nei limiti previsti dalla legge. 